International Journal of Computer Science \& Engineering Survey (IJCSES) Vol.3, No.6, December 2012

\title{
TECHNIQUES AND METHODOLOGIES FOR RECOGNITION OF TAMIL TYPEWRITTEN AND HANDWRITTEN CHARACTERS: A SURVEY
}

\author{
Dr. C P Sumathi ${ }^{1}$ and S Karpagavalli ${ }^{2}$ \\ ${ }^{1}$ Associate Professor, S. D. N. B Vaishnav College for Women, Chennai, Tamil Nadu, \\ India \\ drcpsumathi@gmail.com \\ ${ }^{2}$ Assistant Professor, S. D. N. B Vaishnav College for Women, Chennai, Tamil Nadu, \\ India \\ karpagavalli.subbulgmail.com
}

\begin{abstract}
Recording information both typewritten and handwritten has always been a common mode of communication and also for documenting purposes in day to day life. Machine recognizing of information both, type and hand written has a good practical significance in various context like, reading notes on electronic devices like Tablets, in scanned documents, like Bank cheques etc. The paper is about various methodologies and techniques that were proposed to recognize Tamil characters over time from scanned documents and other similar medium.
\end{abstract}

\section{KEYWORDS}

Tamil character recognition, Techniques, Typewritten, Handwritten, SVM, Neural Networks.

\section{INTRODUCTION}

\subsection{Character Recognition}

The field of Document Analysis and Recognition [7] is vast and used in business oriented applications like automatic cheque processing, information extraction from forms and invoices, automatic document organization and classification etc., and user ori ented applications like OCR systems[1][2]. Character recognition is one of the applications of Document Analysis and Recognition where OCR has gained a momentum since the need for digitization or converting the scanned images of machine printed or hand written text has significant importance in various applications like. Typewritten characters and Handwriting recognition is of great importance in data and word processing, like automated postal and ZIP code reading, acquisition of amount in bank cheques, processing of institutional records and more.

\subsection{Typewritten Vs Handwritten}

Compared to Typewritten, Handwriting recognition is very challenging since character structure and orientation depends on various factors of the persons who write it. Further the field of Handwriting recognition is divided into off-line and on-line recognition, where in off-line recognition, only the image of the handwriting is available for the computer, while in the on-line 
International Journal of Computer Science \& Engineering Survey (IJCSES) Vol.3, No.6, December 2012

case temporal information such as pen tip coordinates as function of time is also available, which made it bit easy compared to the former. Typical data acquisition devices for off-line and on-line recognition are scanners and digitizing tablets, respectively. Again due to the lack of temporal information, off-line handwriting recognition is considered more difficult than on-line. Furthermore, it is also clear that the off-line case is the one that corresponds to the conventional writing task performed by human[8].

\subsection{Tamil Language}

Tamil, one of the oldest languages in the world, is a Dravidian language which is widely spoken in most part of the southern India. It has the official status in the Indian state of Tamil Nadu and in Indian Union territory of Puduchery. Tamil is also an official language of Sri Lanka and Singapore.

It is also spoken by significant minorities in Malaysia and Mauritius.

Tamil orthography is composed of 12 vowels and 18 consonants and one special literal(AK), and each of these sounds is represented by an unique letter. Alphabet system of Tamil language is believed to be a derivation from the ancient Brahmi script which serves as a source for most of the Indian languages. The 12 vowels and the 18 consonants are combined together to form composite letters that are represented by a composite form of the consonants and a secondary symbol for each vowel. In most of the cases, a secondary symbol is added either before or after the consonant to make up the syllable, but in few cases the consonant is slightly modified to form a new shape. Thus, the combination of the vowels and consonants form together of 216 different characters, putting altogether of 247 characters in total.

\subsection{The Alphabet Set}

The vowels and consonants of Tamil alphabet set is given.

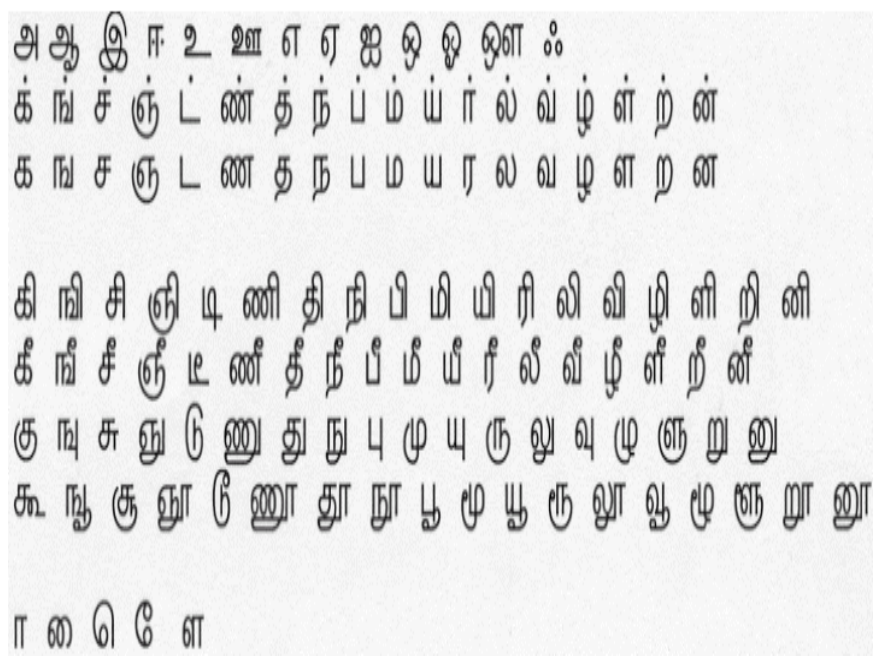

Figure 1. Tamil Alphabet set 


\subsection{Scope and Discussion}

The paper is organized as follows. Section 2 briefs about the various techniques adopted by different authors to recognize Tamil characters over time. Section 3 shows a performance analysis of the discussed techniques.

\section{TECHNIQUES AND METHODOLOGIES}

CR Systems extensively use the methodologies of pattern recognition, which assigns an unknown sample into a predefined class. Numerous techniques for CR can be investigated in four general approaches of Pattern Recognition [28].

- Template Matching

- Statistical Techniques

- Structural Techniques

- Neural Networks

The above approaches are neither necessarily independent nor disjoint from each other. Occasionally, a CR technique in one approach can also be considered to be a member of other approaches.

\subsection{Template Matching}

This is one of the simplest way of character matching the stored prototypes against the character or word to be recognized. Simply, the matching operation determines the degree of similarity between two vectors (group of pixels, shapes, curvature etc.,) in the feature space.

\subsubsection{Direct Matching}

A gray-level or binary input character is directly compared to a standard set of stored prototypes. A template matcher can combine multiple information sources, including match strength and knearest neighbor measurements from different metrics[29][30]. Although direct matching method is intuitive and has a solid mathematical background, the recognition rate of this method is very sensitive to noise.

Siromoney [22] described a method of recognition of Tamil printed characters using an encoded character string dictionary. The scheme employs string features extracted by row and column wise scanning of character matrix. The features in each row are encoded suitably depending upon the complexity of the script to be recognized. A given text is presented symbol by symbol and information from each symbol is extracted in the form of string and compared with strings in the dictionary. When there is an agreement the letters are recognized and printed out in Roman letters following a special method of transliteration. 
International Journal of Computer Science \& Engineering Survey (IJCSES) Vol.3, No.6, December 2012

\subsubsection{Relaxation Matching}

Chinnuswamy [23] proposed an approach for hand written Tamil character recognition. The characters are assumed to be composed of line like elements called primitives, satisfying certain relational constraints. Labeled graphs are used to describe the structural composition of characters in terms of the primitives and the relational constraints satisfied by them. The recognition procedure consists of converting the input image into labeled graph representing the input character and computing correlation coefficients with the labeled graphs stored for a set of basic symbols. This algorithm uses topological matching procedure to compute the correlation coefficients and then maximizes the correlation coefficient.

\subsubsection{Deformable Templates and Elastic Matching}

Elastic Matching technique is used both for offline and online recognition systems.

Prasanth et. al[10] used Dynamic Time Wrapping, a famous Elastic Matching algorithm to recognize online Tamil characters. It is a technique that finds optimal alignment between two time series, if one Time series can be wrapped non-linearly by stretching or shrinking it, along its time axis. This wrapping between two Time series can be used to find the similarity between them. The method had been made more efficient and faster by using Sakoe-Chiba band constraint.

The different feature sets used for classification are

- $\quad x-y$ features ( $x$ and y coordinates of the characters)

- Shape Context(SC) feature combined with the Tangent Angle(TA) feature

- Generalized Shape Context(GSC) feature

- Normalized first and second derivatives with respect to $\mathrm{x}$ and $\mathrm{y}$ and Curvature features.

The above mentioned features form four sets which were used for experimentation.

First set $-\mathrm{x}-\mathrm{y}$ features alone

Second set - SC combined with TA

Third set - GSC alone

Fourth set -7 features viz. the preprocessed $x-y$ features, the normalized first and second derivatives and the curvature. (Local features L7)

Comparing results of the DTW with 4 different sets of features, the SC and TA features are found to be slow. GSC feature is better than SC+TA in terms of accuracy. Finally the local 7 features were the best features in terms of accuracy and speed.

\begin{tabular}{|l|c|r|l|}
\hline \multirow{2}{*}{\begin{tabular}{l}
\multirow{2}{*}{$\begin{array}{l}\text { Feature } \\
\text { set }\end{array}$} \\
Xyn
\end{tabular}} & \multicolumn{2}{|c|}{ Accuracies (\%) } & \multirow{2}{*}{$\begin{array}{l}\text { Time (secs } \\
\text { /symbol) }\end{array}$} \\
\cline { 2 - 3 } X-Y & Top1 & Top2 & \\
\hline SC+TA & 77.65 & 91.13 & 0.5 \\
\hline
\end{tabular}


International Journal of Computer Science \& Engineering Survey (IJCSES) Vol.3, No.6, December 2012

\begin{tabular}{|l|l|l|l|}
\hline GSC & 79.37 & 91 & 6 \\
\hline L7 & 82.37 & 93.65 & 1 \\
\hline
\end{tabular}

Table 1: Accuracies for different feature set

Niranjan et. al [13] compares the experiments of two different approaches like subspace method and DTW on recognizing online Tamil characters.

The Subspace based classification is essentially a linear transformation of the feature space. By selecting the principal directions in which variance is significant, the feature space can be approximated by a lower order space. Each character class is modeled as a subspace. When a core pattern and its variations are found, all the linear combinations of these patterns are treated as members of the class.

DTW is a elastic matching technique, and allows non-linear alignment of sequences and computes a more sophisticated similarity measure, which is especially useful to compare patterns in which rate of progression varies non-linearly which makes similarity measure such as Eucildean distance and cross-correlation unusable.

The performance of both the methods is compared for three different modes

1. Writer Independent(WI),

2. Writer Dependent(WA) and

3. Writer Adaptive(WA).

Comparing the performances of DTW and the Subspace method, we have three different interpretations of errors as follows.

Group1: Errors common for both methods

Group2: Errors specific to subspace

Group3: Errors specific to DTW

\begin{tabular}{|l|l|l|l|l|l|}
\hline Mode & PCA & DTW & Group1 & Group2 & Group3 \\
\hline WI & 11.15 & 8.52 & 3.56 & 7.59 & 4.96 \\
\hline WD & 5.23 & 3.30 & 1.47 & 3.76 & 1.83 \\
\hline WA & 5.41 & 3.60 & 1.20 & 4.21 & 2.40 \\
\hline
\end{tabular}

Table 2: Performances of 3 different modes

Although the performance of DTW based methods is marginally better, in terms of speed, subspace based methods seems to be good.

\subsection{Statistical Techniques}

Statistical decision theory is concerned with the statistical decision functions and a set of optimality criteria, which maximizes the probability of the observed pattern, given the model of certain class [39]. 
International Journal of Computer Science \& Engineering Survey (IJCSES) Vol.3, No.6, December 2012

\subsubsection{Hidden Markov Model (HMM)}

HMM is the most widely and successfully used technique for handwritten character recognition problem [31] [32] [33] [34] [35]. Since it is a stochastic model, HMM can cope with noise and variations in the handwriting.

Bharath et. al. [11] proposed a data-driven HMM-based online handwritten word recognition System for Tamil characters.

Preprocessing of the written characters involves two steps: noise elimination and normalization.

After preprocessing, the features like

- Normalized Y

- Normalized Derivatives

- Angle Features

- Pen-up/Pen-down Bit

are extracted from the symbols.

Then HMM is used build two word models: 1. Symbol modeling and 2. Pen-up Stroke modeling.

1. Symbol modeling - For modeling each and every symbol captured, a simple left-to-right topology with no state skipping was adopted. Then it is trained using Baum-Welch re-estimation procedure. The number of states per model was determined based on the shape complexity of the symbol.

2. Pen-up stroke modeling - The pen-up strokes within a symbol were implicitly modeled using the symbol models whereas the pen-up strokes between symbols were modeled explicitly. The common pen-up stroke models were built which were shared between any pair of symbols and were determined by clustering the inter symbol pen-up strokes obtained from the word samples. The samples falling into each cluster are used to train a two-state left-to-right pen-up HMM having 2 Gaussians per state. For a given word in the lexicon, its word model was built by concatenating the constituent symbol models and having the parallel network of pen-up models inserted in between them. A lexicon was represented as a network of word HMMs where each path in the network from the start node to the final node corresponds to a word. The best path evaluation was determined by the standard Viterbi decoding.

The evaluation of the word recognition system was carried out on different lexicon sizes such as $1 \mathrm{~K}, 2 \mathrm{~K}, 5 \mathrm{~K}, 10 \mathrm{~K}$ and $20 \mathrm{~K}$ to assess the performance.

\begin{tabular}{|l|l|}
\hline Lexicon Size & Accuracy $\%$ \\
\hline $1 \mathrm{~K}$ & 97.96 \\
\hline $2 \mathrm{~K}$ & 95.82 \\
\hline $5 \mathrm{~K}$ & 94.49 \\
\hline $10 \mathrm{~K}$ & 93.17 \\
\hline $20 \mathrm{~K}$ & 92.15 \\
\hline
\end{tabular}

Table 3: Performance accuracy for different Lexicon sizes

In [8] Jagadeesh Kannan et. al. utilizes discrete Hidden Markov Models for recognizing off-line 
International Journal of Computer Science \& Engineering Survey (IJCSES) Vol.3, No.6, December 2012

cursive handwritten Tamil characters. Two HMMs are created for every character, one for modeling the horizontal information and the other for modeling the vertical information respectively.

The maximum likelihood parameter estimation for HMM is obtained by the iterative procedure, the Baum-Welch algorithm [36], with multiple observation sequences.

The discrete Hidden Markov characters Model are trained using standard procedures, and the numbers of state for all the character HMMs are fixed and no skip states are allowed. Two log probabilities for each candidate are calculated using the horizontal direction HMM. Then, the log probabilities are added together to obtain a final 3-best character recognition.

The proposed system was tested with many handwritten documents of different individuals, Olaichuvadi [37] samples, Machine printed, Scanned documents, which yield the following results.

\begin{tabular}{|l|l|}
\hline Count of Words & Efficiency (\%) \\
\hline 25 & 96.4 \\
\hline 50 & 95.6 \\
\hline 100 & 94.8 \\
\hline 150 & 93.7 \\
\hline 200 & 93.0 \\
\hline
\end{tabular}

Table 4 : Efficiency Table

\subsubsection{Non-probabilistic Models}

Support Vector Machines is a training algorithm for learning, classification and regression rules from data. It is one of the efficient learning methodology in Aritificial Intelligence.

SVM is a Non-probabilistic model that includes the following components:

- Regularized linear learning models (such as classification and regression),

- Theoretical bounds,

- Convex duality and the associated dual-kernel representation, and

- Sparseness of the dual-kernel representation.

This makes SVMs and related kernel-based learning methods special and interesting. SVMs have also successfully been applied in practice, especially for classification problems. Although many problems that have been successfully solved by SVMs could also have been solved successfully by standard statistical methods.

In [15] Seethalakshmi et. al. develops an OCR that refers to the process of converting printed Tamil text documents into software translated Unicode [38] Tamil text.

Classification is done using the features such as

- height and width of the character 
International Journal of Computer Science \& Engineering Survey (IJCSES) Vol.3, No.6, December 2012

- horizontal lines-short and long

- vertical lines-short and long

- number of circles

- number of horizontally oriented and vertically oriented arcs

- centroid of the image etc.,

are extracted that corresponds to each character glyph.

The classification is done by Support Vector Machines. The kernel function used was $\mathrm{RBF}$ (Radial Basis Function) which is far by the most popular choice. SVM consists of a learning module and a classification module. The training model takes the input file, target file and trains the network. In the classification model, the various class labels like class 1, 2, 3, .... 247 are given. Thus the SVM learns and produces correct labels of the classes. After classification the characters are recognized and a mapping table is created in which the Unicodes [36] for the corresponding characters are mapped. The scanned image which was through various function blocks is finally compared with the recognition details from the mapping table from which corresponding Unicodes are accessed and printed using Unicode fonts so that the OCR is achieved.

In [25] Shanthi et. al. described a recognition system for offline unconstrained handwritten Tamil characters based on support vector machine(SVM). SVM is a type of classifier based on a novel statistical learning technique. Due to the difficulty in great variation among handwritten characters, the system is trained with 106 characters and tested for 34 selected Tamil characters. The data set is chosen such that the sample data set represents almost all the characters. Data samples are collected from different writers on A4 sized documents. They are scanned using a flat bed scanner at a resolution of $300 \mathrm{dpi}$ and stored as grey scale images. Various pre processing operations are preformed on the digitized image to enhance the quality of the image. Random sized pre processed image is normalized to uniform sized image. Pixel densities are calculated for different zones of the image and these values are used as the features of a character. These features are used to train and test the support vector machine.

Shivsubramani et. al. [14] presented a method for recognizing printed Tamil characters exploring the inter relationship between the alphabets. Multi-class Hierarchical Support Vector Machine, a variant of Multi-class Support Vector Machine is for recognition.

Here the individual characters are examined and a lot of inter-class dependencies were identified based on their shapes, which enables the characters to be organized into hierarchies for recognizing the characters.

The feature values of similar characters have a very minimal difference. The characters exhibiting similarity were organized into hierarchies for classification. A character feature is found to exhibit the property of a particular subclass and the characters not belonging to this hierarchy need not be considered for classification. Thereby a 126 class classification problem is broken down into 10 class or 8 class problem. It has been found to be efficient than Multi-Layer Perceptron(MLP), K-Nearest neighbor(KNN) and Decision Trees(DT).

Jagadeesh et. al [21] presented a system that fuses two algorithms for recognition of Tamil characters. The advantage and efficiency of two algorithms are fused to increase accuracy. 
International Journal of Computer Science \& Engineering Survey (IJCSES) Vol.3, No.6, December 2012

After the pre processing stage, the characters are recognized using a fusion of HMM and SVM. Finally a Radial Basis Functional Neural network was trained with the output of both the algorithms. If HMM or SVM gives a false character, the neural network is trained with the weight age of both the algorithms and the actual character.

The idea behind this fusion is

1. if one algorithm fails to identify character another algorithm may support in identifying the character.

2. if one algorithm gives wrong character another may give a correct one.

3. the possibility for same wrong identification by both the algorithm is less.

4. if one algorithm gives wrong result, the decision of choosing the correct result is done by neural network.

This process is done for all the possible false recognition of the two algorithms. When both the algorithms not giving same character, trained RBFNN is used to retrieve the actual character. Maximum of 250 Thirukkurals were chosen for testing the efficiency of the system.

\subsubsection{Fuzzy Set Reasoning}

Suresh et. al. [24] describes an approach to use the fuzzy concept on handwritten Tamil characters to classify them as one among the prototype characters using a feature called distance from the frame and a suitable membership function. The unknown and prototype characters are preprocessed and considered for recognition. The theory of fuzzy set provides an approximate but effective means of describing the behavior of ill-defined systems. Patterns of human origin like handwritten characters are to some extent found to be fuzzy in nature. It is described to use fuzzy conceptual approach effectively. The algorithm is tested for about 250 samples for numerals and seven chosen Tamil characters and the success rate obtained varies

from $76 \%$ to $94 \%$.

\subsection{Structural Techniques}

Jagadeesh et. al [9] utilizes Octal graph conversion for recognizing offline Tamil characters. The proposed approach ventures a solution for recognition, which converts the letter written into an octal graph, by representing each pixel of the given character as a node of a graph. Each node has eight fields so termed as octal graph. The graph tries to represent the basic form of a letter independent of the style of writing. Using the weights of the graphs and by the appropriate feature matching with the predefined characters, the written characters are recognized.

An octal graph unlike a normal graph has a node with eight pointers and a data field. Based on the neighboring pixels the pointer values are assigned to the various fields of the octal node. These octal nodes are connected to the other nodes based on the threshold value. The figure shows the Octal Node representation of a Tamil Vowel.

\subsection{Neural Networks}

A neural network is defined as a computing architecture that consists of massively parallel interconnection of adaptive 'neural' processors. Because of its adaptive nature, it can adapt to 
International Journal of Computer Science \& Engineering Survey (IJCSES) Vol.3, No.6, December 2012

changes in the data and learn the characteristics of input signal. A Neural network has many nodes. The output from one node is fed to another one in the network and the final decision depends on the complex interaction of all the nodes. In spite of the different underlying principles, it can be shown that most of the neural network architectures are equivalent to statistical pattern recognition methods[38].

Aparna et. al [19] presents an OCR system for Tamil newsprint. ANN is used for solving key problems of segmentation and for character recognition.

The system train a Radial basis function neural network for character recognition. All the characters including the vowels, consonants, the special character or the Grantha letters and the English numerals 0 to 9 and the punctuation marks making a total of 157 characters are taken for training the network. The characters are placed at the center of $52 \times 52$ window and the input patters to be trained by the RBF neural network are obtained by taking the dot product of the character with each of the 40 Gabor filters with 10 along each of four directions. The RBF neural network has 157 outputs each output corresponding to an alphabet.

This trained neural network is used for the recognition of segmented characters which yield 85 to 90 percent of accuracy, in general.

Sudha et. al. [26] describes an approach to recognize handwritten Tamil characters using a Multilayer perception with one hidden layer. The feature extracted from the handwritten character is Fourier descriptors. Also an analysis was carried out to determine the number of hidden layer nodes to achieve high performance of back propagation network in the recognition of handwritten Tamil characters.

The system was trained using several different forms of handwriting provided by both male and female participants of different age groups. test results indicate that Fourier descriptors combined with back propagation network provide good recognition accuracy of $97 \%$ for handwritten Tamil characters.

\section{Performance Analysis}

The paper gives an overview of the main approaches that where used in the ongoing research in the Tamil character recognition field. Though each of the above discussed methods has their own superiorities and drawbacks, the recognition accuracy rates are reported to be above $85 \%$. However, it is very difficult to compare the success of each of the methods, especially in terms of recognition rates, due to various factors like, different database, constraints and sample spaces.

The ongoing research is successful on texts that are discrete and neat on a high quality paper or on the Tablet, whose recognition rates are above $85 \%$, and a few research was on cursive offline texts. For handwritten texts that are poor on a low quality paper, there is still a need of intensive research.

Most of the offline texts are recognized using HMMs and Neural Networks. It is also better to combine different techniques, since the best of each technique is used for 
International Journal of Computer Science \& Engineering Survey (IJCSES) Vol.3, No.6, December 2012 recognition which may improve the accuracy rates.

\section{CONCLUSION}

The idea of the paper is to give an overview of the ongoing research in optical character recognition systems for Tamil characters, both online and offline Handwritten and Typewritten characters. The performance of various methods has also been analyzed, which gives a picture of efficient factor of each method.

\section{REFERENCES}

[1] Mantas, J., 1986. "An Overview of character recognition methodologies", Pattern Recognition, 19(6):425-430.

[2] Govindan V. K and A. P Shivprasad, 1990. "Character Recognition- A Review", Pattern Recognition, 23(7):671-683.

[3] Pal U and B B Chaudhuri, 2004."Indian Script character recognition: A Survey", Pattern Recognition, 37:1887-1899.

[4] R Plamondon and S n Srihari, 2000. "Online and Offline handwritten recognition: A Comprehensive survey", IEEE transactions on PAMI Vol. 22(1), pp 63-84.

[5] Hewavitharana, S and H. C. Fernando, 2002. "A two-stage classification approach to Tamil handwriting recognition".

[6] N. Shanthi and K. Duraiswamy, 2005. "Preprocessing algorithms for the recognition of Tamil handwritten characters"

[7] R. Jagadeesh Kannan, R. Prabhakar, 2009. "A Comparative study of Optical Character Recognition for Tamil script".

[8] R. Jagadeesh Kannan, R. Prabhakar, 2008. "Off-Line cursive handwritten Tamil character recognition".

[9] R. Jagadeesh Kannan, R. Prabhakar, 2008. "An improved Handwritten Tamil character recognition system using Octal Graph".

[10] L. Prasanth, V. Jagadeesh Babu, R.Raghunath Sharma, G. V. Prabhakara Rao. "Elastic matching of Online handwritten Tamil and Telugu scripts using local features".

[11] Bharath A, Sriganesh Madhvanath, 2007. "Hidden Markov Models for online handwritten Tamil word recognition".

[12] Niranjan Joshi, G. Sita and A. G. Ramakrishnan. "Compariso of Elastic matching algorithms for online Tamil handwritten character recognition".

[13] Niranjan joshi, G. Sita, A. G. Ramakrishnan. "Tamil handwriting recognition using subspace and DTW based classifiers". 
International Journal of Computer Science \& Engineering Survey (IJCSES) Vol.3, No.6, December 2012

[14] Shivsubramani K, Loganathan R, Srinivasan C J, Ajay V, Soman K P, 2007. "Multiclass Hierarchical SVM for recognition of printed Tamil characters".

[15] Seethalakshmi R, Sreeranjani T R, Balachandar T. "Optical Character Recognition for printed Tamil text using Unicode".

[16] Sandhya Arora, Debotosh Bhattacharjee, Mita Nasipuri, 2008. "Combining Multiple feature extraction techniques for handwritten Devnagari character recognition".

[17] C. S Sundaresan, S. S Keerthi. " A study representation for Pen based handwriting recognition of Tamil characters".

[18] K. H Aparna, Vidhya Subramanian, M. Kasirajan, G. Vijay Prakash, V. S Chakravathy, 2004. "Online handwriting recognition of Tamil".

[19] K. H Aparna, Sumanth Jaganathan, P. Krishnan, V. S Chakravathy. "Document Image Analysis with specific application to Tamil Newsprint".

[20] Deepu V, Sriganesh M, Ramakrishnan A G. "Principal Component Analysis for Online handwritten character recognition".

[21] R. Jagadeesh Kannan, R. Prabhakar, 2008. "Accuracy Augmentation of Tamil OCR using algorithm Fusion".

[22] Siromoney et al., 1978. "Computer recognition of printed Tamil character", Pattern Recognition $10: 243-247$.

[23] Chinnuswamy. P and S G Krishnamoorthy 1980. "Recognition of hand printed Tamil characters", Pattern Recognition, 12:141-152.

[24] Suresh et al., 1999. "Recognition of hand printed Tamil characters using classification approach". ICAPRDT’99, pp:63-84.

[25] N Shanthi and K Duraiswamy, "Performance comparison of different Image sizes for recognizing Unconstrained handwritten Tamil characters using SVM".

[26] Sutha, J Ramaraj, N Sethu. "Neural Network based Offline Tamil handwritten character recognition system".

[27] Nafiz Arica, T. Yarman-Vural, "An Overview of Character Recognition focused on Off-line Handwritting".

[28] A. K. Jain, R. P. W. Duin, J. Mao "Statistical Pattern Recognition: A Review", IEEE Trans. Pattern Analysis and Machine Intelligence, vol 22, no 1, pp. 4-38, 2000.

[29] D. Tubbs, "A note on Binary Template matching", pattern Recognition, vol. 22, no. 4, pp 359-365, 1989.

[30] P. D. Gader, B. Forester, M. Ganzberger, A. Gillies, B. Mitchell, M. Whalen and T. Youcum, "Recognition of handwritten digits using Template and Model matching", Pattern Recognition, vol. 24, no.5, pp 421-431, 1991.

[31] M. A. Mohamed, P. Gader, "Genearlized Hidden Markov Models-Part II: Application to Handwritten Word Recognition”, IEEE Trans. Fuzzy Systems, Vol.8, no.1, pp. 82-95, 2000. 
International Journal of Computer Science \& Engineering Survey (IJCSES) Vol.3, No.6, December 2012

[32] M. A. Mohamed, P. Gader, "Handwritten Word Recognition using Segmentation-free Hidden Markov modeling and Segmentation based Dynamic Programming techniques", IEEE Trans. Pattern Analysis and Machine Intelligence, Vol.8, no.5, pp. 548-554, 1996.

[33] A. Kornai, K. M. Mohiuddin, S. D. Conell, "An HMM-Based legal amount field OCR system for checks”, IEEE Trans, Systems, Man nd Cybernetics. pp 2800-2805, 1995.

[34] M. Y. Chen, A. Kundu, J. Zhou, "Offline handwritten word recognition using Hidden Markov Model type stochastic network", IEEE Trans. Pattern Recognition and Machine Intelligence, vol 16, pp 481-496, 1994.

[35] M. Y. Chen, A. Kundu, S. N. Srihari, "Variable Duration Hidden Marokov Model and Morphological segmentation for handwritten word recognition", IEEE Trans. Image Processing, vol 4, pp 1675-1688, 1995.

[36] K. Khatatneh, "Probabilistic Artificial Neural Network for recognizing the Arabic handwritten characters", Journal of Computer Science 3(12),881-886,2006.

[37] unicode.org/charts/PDF/U0B80.pdf

[38] Jacek M Zurada, Introduction to Artificial Neural Systems

[39] P. A. Devijier, J. Kittler, Pattern Recognition,: A Statistical Approach, Prentice Hall, 1982. 